\title{
Covid-19 and Patients with IBD: Who Is at Highest Risk for Severe Complications?
}

\author{
Sara Horst ${ }^{1}$ \\ Accepted: 8 June 2021 / Published online: 28 June 2021 \\ (c) The Author(s), under exclusive licence to Springer Science+Business Media, LLC, part of Springer Nature 2021
}

During the year 2020, GI physicians were faced with many questions, in particular how patients with inflammatory bowel disease (IBD) would be affected by infection with the severe acute respiratory syndrome coronavirus 2 (SARS$\mathrm{CoV}-2$ ), the cause of coronavirus disease 2019 (COVID19). Research teams and organizations rapidly led intense efforts to quickly advance the understanding of the impact of COVID-19 for patients with IBD, including the formation of the Surveillance Epidemiology of Coronavirus Under Research Exclusion for Inflammatory Bowel Disease database (SECURE-IBD). This voluntary international registry enabled providers to gather and disseminate information about patients with IBD who developed a COVID-19 infection [1].

Early data was reassuring, showing that the risk of mortality for patients with IBD from COVID-19 was not different from that of the general population. Other studies suggested that patients with IBD do not have an increased risk of COVID-19 infection compared with the general population $[2,3]$. The first analysis of patients in the SECURE-IBD registry identified several risk factors for severe complications (hospitalization, need for ICU care, or death) from COVID-19, included increasing age, prednisone use, and number of comorbidities. Age and number of comorbidities also increases the risk of severe COVID-19 in the general population. Reassuringly, biologic medication use did not increase the risk of severe complications from COVID-19 $[1,4]$. In addition to corticosteroid use, thiopurine use or thiopurine use in combination with a biologic may increase the risk of severe complications from COVID-19 such as hospitalization [5].

As the COVID-19 pandemic has progressed, understanding the relative risks of specific comorbidities has become

Sara Horst

sara.n.horst@vumc.org

1 Division of Gastroenterology, Hepatology, and Nutrition, Vanderbilt University Medical Center, Nashville, USA even more important. In this issue of Digestive Diseases and Sciences, Parekh A. et al. [6] provide a detailed analysis of comorbidities and their effect on severe COVID-19 disease. This study evaluated patient data from the SECUREIBD registry up until November 2020, including 2,035 patients with IBD who developed COVID-19, originating from patients in 58 countries. Five hundred and thirty-eight (26.4\%) experienced hospitalization or death, and 64 (3.1\%) died. Detailed evaluation of the contribution of specific comorbidities to this risk was evaluated with logistic regression analysis. Increasing age and corticosteroid use remained significantly associated with severe complications, as has been shown in prior work $[1,7]$.

In evaluating specific comorbidities in a composite model, several conditions increased the likelihood of severe complications from COVID, including asthma, diabetes, chronic liver disease, hypertension, and chronic lung disease. Comorbid conditions with the highest odds of complications included patients with chronic kidney disease (CKD) and chronic obstructive pulmonary disease (COPD). Most of these comorbidities that increased the risk of severe COVID19 complications was confirmed in a recent national healthcare database study evaluating 196,403 patients with IBD and COVID-19 infection [4]. Interestingly, in the study by Parekh et al. [6], a history of prior cancer or cardiovascular disease did not contribute to increased risk of complications from COVID-19. Nevertheless, only the presence or absence of these comorbidities in the patient history was noted by the provider on the registry questionnaire. A "cancer history" for a patient can vary broadly, ranging from a long-term ( $>10$ year) remission to a current advanced tumor with widespread metastases; this imprecision is therefore a limitation of this evaluation.

One important aspect to highlight in the Parekh et al. study is the significant rate of hospitalizations or death (26.4\%) from COVID-19 for patients with IBD. Given the voluntary nature of data input, it is possible that providers only entered patients with more severe disease into the 
registry or may not even have been alerted if their patients had very mild symptoms. This could lead to higher numbers of reported COVID-19 complications than a true population-based cohort. Nonetheless, similar hospitalization rates from COVID-19 were reported from Italy for patients with IBD, indicating a $28 \%$ hospitalization rate and $8 \%$ mortality rate [7]. A metanalysis found a $31 \%$ hospitalization rate in patients with IBD who developed COVID-19, with a $3.8 \%$ mortality rate [8]. In the nationwide US database study referenced earlier, hospitalization rates were again similar (24.14\%). These rates were similar to patients who did not have IBD when controlling for comorbid conditions [4].

Hospitalization rates for COVID-19 patients in general are exceedingly high for a viral illness. Since far more information is available now than at the beginning of the pandemic regarding the risk of complications from COVID19, patients at highest risk, including those with CKD or COPD, are at utmost priority for vaccination. Although ever evolving, data for vaccines against SARS-CoV-2 continue to validate their safety and very rare significant adverse events [9]. There is some concern that patients with IBD on immunosuppression may have an inferior vaccine response. A recent study evaluated 27 patients with IBD receiving biologic therapy with or without immunomodulator medication reported high rates of seroconversion $(85 \%$; $23 / 27$ pts; antiSARS-CoV-2 anti-spike protein receptor-binding protein antibodies) who had a course of two Pfizer BN162b2 vaccine [10], a reassuring observation. More research is underway to understand vaccine response in the IBD patient population. The bottom line is that the medical community should continue to reach out, especially to the highest risk patients with IBD, to discuss strategies including vaccination to minimize the future impact of COVID-19 complications.

\section{Key Message}

- The SECURE-IBD registry provided valuable data useful in defining risk of severe COVID-19 in IBD patients.

- Age and steroid use increase the risk of COVID-19

- Patients with comorbidities, especially kidney disease and COPD, may at the highest risk for morbid COVID19 outcomes.

\section{References}

1. Brenner EJ, Ungaro RC, Gearry RB et al. Corticosteroids, but not TNF antagonists, are associated with adverse COVID-19 outcomes in patients with inflammatory bowel diseases: results from an international registry. Gastroenterology. 2020;159:481$491 \mathrm{e} 483$.

2. Aziz M, Fatima R, Haghbin H, Lee-Smith W, Nawras A. The incidence and outcomes of COVID-19 in IBD patients: a rapid review and meta-analysis. Inflamm Bowel Dis. 2020;26:e132-e133.

3. Khan N, Patel D, Xie D, Pernes T, Lewis J, Yang YX. Are patients with inflammatory bowel disease at an increased risk of developing SARS-CoV-2 than patients without inflammatory bowel disease? results from a nationwide veterans' affairs cohort study. Am J Gastroenterol. 2021;116:808-810.

4. Singh S, Khan A, Chowdhry M, Bilal M, Kochhar GS, Clarke K. Risk of severe coronavirus disease 2019 in patients with inflammatory bowel disease in the United States: a multicenter research network study. Gastroenterology. 2020;159:1575-1578 e1574.

5. Ungaro RC, Brenner EJ, Gearry RB et al. Effect of IBD medications on COVID-19 outcomes: results from an international registry. Gut. 2021;70:725-732.

6. Parekh, R, Zhang, X, Ungaro, RC, et al. Presence of comorbidities associated with severe coronavirus infection in patients with inflammatory bowel disease. Dig Dis Sci. (Epub ahead of print). https://doi.org/10.1007/s10620-021-07104-0.

7. Bezzio C, Saibeni S, Variola A et al. Outcomes of COVID19 in 79 patients with IBD in Italy: an IG-IBD study. Gut. 2020;69:1213-1217.

8. D'Amico F, Danese S, Peyrin-Biroulet L. Systematic review on inflammatory bowel disease patients with coronavirus disease 2019: it is time to take stock. Clin Gastroenterol Hepatol. 2020;18:2689-2700.

9. CDC: COVID-19 Vaccine Safety Technical (VaST) Work Group Reports. Control CoD. https://www.cdc.gov/vaccines/acip/workgroups-vast/technical-report.html. Published 2021. Accessed 05/24/2021.

10. Kennedy NA, Lin S, Goodhand JR, et al. Infliximab is associated with attenuated immunogenicity to BNT162b2 and ChAdOx1 nCoV-19 SARS-CoV-2 vaccines in patients with IBD. Gut. 2021. https://doi.org/10.1136/gutjnl-2021-324789.

Publisher's Note Springer Nature remains neutral with regard to jurisdictional claims in published maps and institutional affiliations. 